\title{
Productividad y rentabilidad del cultivo de camarones marinos en el Golfo de Nicoya, Costa Rica
}

\section{Productivity and profitability of shrimp mariculture in the Gulf of Nicoya, Costa Rica}

\author{
José A. Valverde-Moya ${ }^{1}$ Jorge Alfaro-Montoya ${ }^{2 *}$
}

\begin{abstract}
RESUMEN
El objetivo del presente estudio es la evaluación de la productividad y la rentabilidad económica del cultivo comercial de camarones marinos en el Golfo de Nicoya, Costa Rica. El estudio se llevó a cabo en el 2013 en fincas comerciales localizadas en los sectores de Abangaritos de Puntarenas, Colorado de Abangares, Puerto Jesús de Nicoya y Lepanto de Puntarenas. Se sembraron 25.4 has en modalidad de 3 ciclos cortos y 24.6 has en modalidad de ciclo largo, siguiendo un plan de manejo convencional. La productividad en $\mathrm{kg} / \mathrm{ha} / \mathrm{año} \mathrm{fue} \mathrm{superior} \mathrm{en} \mathrm{los} 3$ ciclos cortos (2 $605 \mathrm{~kg} / \mathrm{ha} / \mathrm{año}$ ) con respecto al ciclo largo (2 $138 \mathrm{~kg} / \mathrm{ha} / \mathrm{año})$. Los costos variables de producción fueron más altos en los ciclos cortos (US\$ 8 389) que en el ciclo largo (US\$ 6 783), debido a la utilización de dos tecnologías distintas de cultivo en cuanto a densidades de siembra, cosechas por año, tamaño del producto, precio en el mercado y recambios de agua. Los ingresos por concepto de ventas al pie de finca fueron más altos en los ciclos cortos (US\$ 14 671/ha) que en el ciclo largo (US\$ 12 281/ha). El estudio de sensibilidad económica sugiere la realización de 3 ciclos cortos en lugar de un solo ciclo largo, debido a la mayor productividad y rentabilidad (US\$ 5 536/ha contra US \$ $4752 /$ ha); sin embargo, la estrategia de producción no debe ser fija sino flexible para ajustarse al cambio, sobre todo del precio del producto en el mercado en esos momentos.
\end{abstract}

Palabras claves: Decapoda, Penaeidae, Litopenaeus vannamei, cultivo comercial, camarones.

\begin{abstract}
The main objective of this study was to evaluate productivity and economic profitability of commercial culture of marine shrimp in the Gulf of Nicoya, Costa Rica. The study was conducted in 2013 in commercial farms located in Abangaritos de Puntarenas, Colorado de Abangares, Puerto Jesús de Nicoya and Lepanto de Puntarenas. A total of 25.4 ha in 3 short cycles and 24.6 ha in one long cycle were used, following a conventional management plan. Productivity in $\mathrm{kg} / \mathrm{ha} /$ year was higher in the 3 short cycles $(2,605 \mathrm{~kg} / \mathrm{ha} /$ year $)$ compared to the long cycle $(2,138$ $\mathrm{kg} / \mathrm{ha} /$ year). Variable costs of production for the short cycles were higher (US\$8,389) than those for the long cycle (US\$6,783), due to the implementation of 2 different culture technologies concerning stocking densities, harvests per year, product size, market price and water exchange. Income from farm sales was higher for the short cycles (US\$14,671/ha) than for the long cycle (US\$12,281/ha). The economic sensitivity analysis suggests the implementation of 3 short cycles rather than one long cycle based on higher productivity and profitability (US\$5,536/ha compared to US $\$ 4,752 /$ ha); however, the production strategy must be flexible to adjust to changes, particularly regarding product market price at any given moment.
\end{abstract}

Keywords: Decapoda, Penaeidae, Litopenaeus vannamei, commercial culture, shrimp.

Consultor en Acuicultura, jvalmo@yahoo.com.

Escuela de Ciencias Biológicas, Universidad Nacional, Heredia, Costa Rica, jorge.alfaro.montoya@una.cr*

Recibido: 10 de marzo de 2014

Corregido: 5 de mayo de 2014

Aceptado: 26 de mayo de 2014

DOI: http://dx.doi.org/10.15359/revmar.6.3

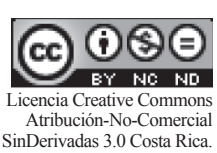

Rev. Mar. Cost. ISSN 1659-455X. Vol. 6: 37-53, Diciembre 2014. 


\section{INTRODUCCIÓN}

La industria del cultivo de camarones marinos en Costa Rica experimentó un auge sin precedentes en el precio del producto en el 2013, debido a cuatro factores fundamentales: la baja producción de camarón en México por una enfermedad que ocasionó altas mortalidades y pérdidas millonarias (Central AmericaData.COM, 2013a), el nuevo arancel a Ecuador por parte de Estados Unidos que representó un aumento en la demanda de camarón (PROCOMER, 2013), el levantamiento del impedimento impuesto por Estados Unidos a la importación de camarón costarricense (CentralAmericaData.COM, 2012; Castillo, 2012) y la prohibición por parte de la Sala Constitucional al otorgamiento de nuevas licencias a las redes de arrastre para la pesca de camarón (CentralAmericaData.COM, 2013b).

El camarón ha sido uno de los productos de mayor dinamismo para las exportaciones del sector pecuario y pesca en Costa Rica. En el 2012 se exportaron alrededor de US\$ 8.5 millones con destino a Europa (Alemania, Holanda y España), Estados Unidos y Japón, y en los primeros siete meses del 2013 ya se habían exportado más de US\$ 3.6 millones. De enero a mayo del mismo año, el camarón estuvo entre los cuatro principales productos del sector pesquero que mostraron crecimiento en sus exportaciones (59.7\%) con respecto al 2012, de acuerdo con datos de PROCOMER (2013). Además, es interesante el hecho de que se han encontrado oportunidades en mercados distintos a los tradicionales como Egipto, China, Rusia y México, que constituyen destinos donde los empresarios costarricenses podrían analizar la búsqueda de nuevos negocios. El camarón es el alimento de origen acuático más popular en Estados Unidos y representa un $25 \%$ del consumo total de este tipo de productos, siendo el precio el factor más determinante para los consumidores de ese país, debido a que consideran que las regulaciones existentes de salud y seguridad proveen un marco adecuado para la calidad de los productos (Castillo, 2012).

En Costa Rica existen suficiente área y niveles adecuados de producción en el cultivo de camarones marinos como para compensar la mayor parte de la extracción con redes de arrastre, reduciendo significativamente sus efectos negativos en el medio natural (Álvarez \& Salazar, 2010). No obstante, el incremento en la producción debe hacerse mediante la implementación de Planes de Manejo y Buenas Prácticas Acuícolas para que reduzcan el impacto potencial de esta actividad al ambiente. En los últimos años, las tecnologías de cultivo se han venido estabilizando, debido a que han permitido obtener de manera continua y predecible niveles adecuados de producción (Valverde-Moya \& AlfaroMontoya, 2013). Según los mismos autores, el sector del Pacífico norte a ambas márgenes del Golfo de Nicoya ha sido uno de los más productivos y se ha caracterizado por realizar estos ciclos de cultivo desde febrero hasta noviembre, dejando un vacío sanitario entre diciembre y enero de cada año. 
En la productividad del cultivo de camarones marinos en estanques de producción influyen directamente la densidad de siembra, la duración del cultivo, el manejo de las fincas y el tamaño del camarón a la cosecha entre los ciclos. En la rentabilidad deben considerarse otros aspectos importantes, como los costos de operación y el precio nacional e internacional del producto en el momento de las cosechas parciales y totales, según el tamaño del camarón. Los costos de producción varían cuando se trata de tecnologías distintas de cultivo y los precios de ventas fluctúan dependiendo de la oferta nacional e internacional del producto.

Ante estas perspectivas y fluctuaciones, Valverde-Moya \& AlfaroMontoya (2013) recomiendan hacer un estudio donde se evalúen la producción, el precio según el tamaño del producto y los costos variables de producción para la toma de la mejor decisión por parte de los productores nacionales en cuanto a la densidad de siembra, la estrategia de producción y de comercialización que deben llevar a cabo, con el fin de lograr su propósito primordial que es obtener ganancias económicas. Por lo tanto, el objetivo del presente estudio fue evaluar la productividad y la rentabilidad económica del cultivo de camarones marinos en estanques de producción en el Golfo de Nicoya, Costa Rica, utilizando dos estrategias distintas de manejo como lo son: 3 ciclos cortos consecutivos o un solo ciclo largo en el transcurso del año.

\section{MATERIALES Y MÉTODOS}

El estudio se llevó a cabo desde febrero hasta diciembre del 2013 en fincas comerciales localizadas en los sectores de Abangaritos de Puntarenas $\left(\mathrm{N}^{\circ}\right.$ 1), Colorado de Abangares $\left(\mathrm{N}^{\circ}\right.$ 2), Puerto Jesús de Nicoya $\left(\mathrm{N}^{\circ} 3\right)$ y Lepanto de Puntarenas $\left(\mathrm{N}^{\circ} 4\right)$ en la región del Pacífico norte de Costa Rica en las márgenes este y oeste del Golfo de Nicoya (Fig. 1). El estudio económico comparó dos estrategias de cultivo: 8 estanques con áreas de 2.2 a 4.2 has en los 3 ciclos cortos correspondiendo a un total de 25.4 has y 8 estanques de 1.2 a 5.0 has para un total de 24.6 has en el ciclo largo. En los ciclos cortos, las siembras se realizaron el 21 de febrero, 7 de junio y 12 de septiembre con densidades de 8.0 a 10.0 camarones/ $\mathrm{m}^{2}$, mientras que en el ciclo largo se sembraron 4 estanques el 13 de marzo y 4 estanques el 10 de abril utilizando una densidad de 15 camarones $/ \mathrm{m}^{2}$.

Los estanques se prepararon después del vacío sanitario entre diciembre y enero, según protocolos convencionales descritos en Valverde-Moya \& Alfaro-Montoya (2013). En resumen, luego de la aplicación de cloro o insecticida, el agua se dejó salir y se volvió a llenar hasta nivel de siembra con agua finamente filtrada en malla marca Nytex ${ }^{\circledR}$ de 700 micras en ambos ciclos. En las fincas de ciclo corto se generó la proliferación del fitoplancton con aplicación de fertilizante inorgánico a base de urea, como se ha hecho rutinariamente. Se sembraron poslarvas 12 (PL12) y el manejo de los estanques fue similar al descrito por los anteriores autores. Los 


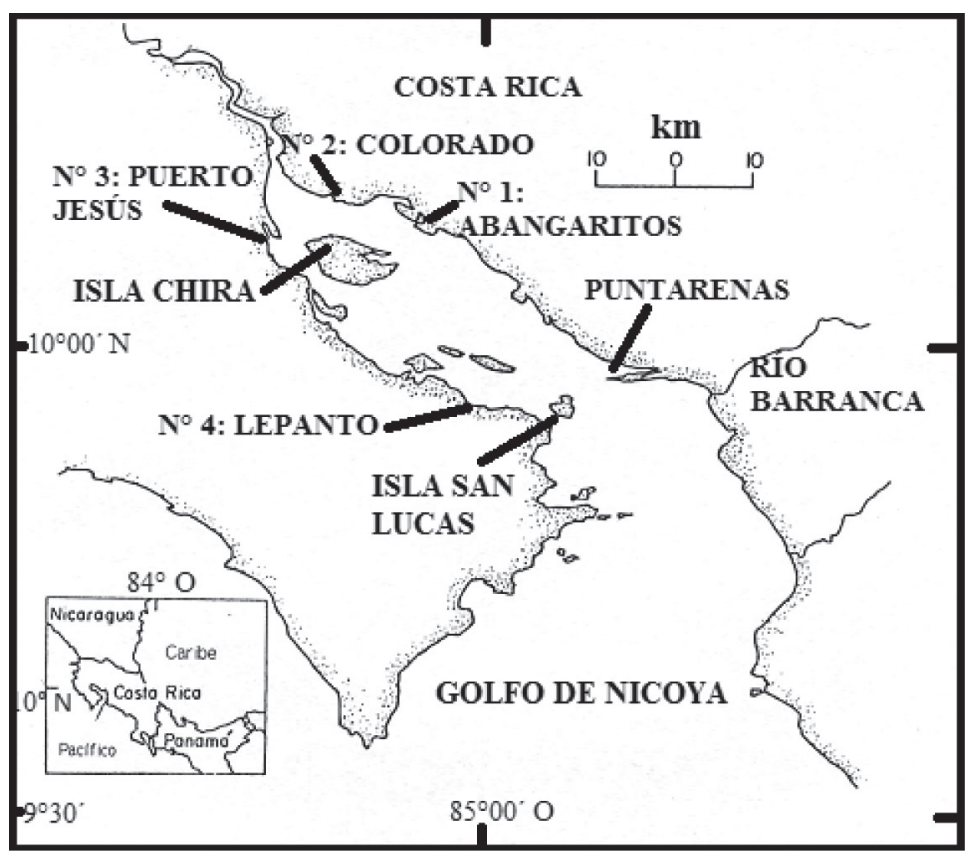

Fig. 1. Localización de las fincas camaroneras en la región del Pacífico norte de Costa Rica, a ambas márgenes del Golfo de Nicoya

Fig. 1. Location of shrimp farms in the Northern Pacific region of Costa Rica, at both margins of the Gulf of Nicoya

regímenes diarios de alimentación fueron de 2 veces (mañana y tarde) en el ciclo corto y 3 veces (mañana, mediodía y tarde) en el ciclo largo.

Se compararon entre ambos tipos de producción, los promedios de sobrevivencia, el crecimiento, la conversión alimenticia y la tasa de alimentación. La productividad se expresó en $\mathrm{kg} / \mathrm{ha} / \mathrm{año}$ en cada tipo de sistema. El rendimiento se calculó tomando en cuenta los kilos producidos por millar de PL's sembradas. El análisis de sensibilidad económica se hizo con base en las ganancias obtenidas de ambos tipos de cultivos. Las ganancias se calcularon tomando en cuenta la biomasa producida $(\mathrm{kg})$ y el precio a un tipo de cambio de 500 colones costarricenses $(\phi)$ por dólar americano US\$ $(\$=\phi 500)$ en ese momento, según el tamaño del producto entero $(\mathrm{g})$ al pie de finca. Los precios en el mercado nacional para camarón pequeño $(<20 \mathrm{~g})$ e internacional para camarón grande ( $>20 \mathrm{~g}$ ) fueron suministrados por la Cámara de Productores Camaroneros (CAPROCAM) del país.

En el cálculo de los costos variables se incluyeron el alimento, la poslarva, la mano de obra y la energía en el bombeo (eléctrica o diésel) como los más importantes. Otros costos menos significativos correspondieron a materiales, equipos en insumos, con el transporte incluido, para el mantenimiento de la finca y la 
preparación de los estanques. Como un costo fijo se incluyeron el pago de impuestos, las pólizas y las afiliaciones.

El costo por concepto de alimento se calculó con base en los registros del gasto diario en la bitácora del biólogo asesor y el precio por saco (\$25/saco de $25 \mathrm{~kg}$ ). El precio de la PL dependió en gran medida de la cantidad suministrada por concepto del costo del flete desde Nicaragua. Dependiendo de la cantidad transportada, este osciló de $\$ 4.80$ a $\$ 5.50$ por millar, puesto al pie de finca con la asesoría en la siembra.

La mano de obra se calculó según el salario semanal de cada empleado, considerando el salario mínimo de $\$ 17.2$ por día de 8 horas laborales para un peón agrícola en labores livianas, según el Ministerio de Trabajo y Seguridad Social (2013). La seguridad se dio por medio de un guarda de noche en cada finca desde que el camarón alcanzó los $7 \mathrm{~g}$ de peso promedio y existió el peligro de robo hasta la cosecha final. El salario del guarda es de $\$ 20.0$ por noche en jornada de 9 hs (de 8 p.m. a 5 a.m.) o $\$ 600$ por mes, corrido similar a lo estipulado por el Ministerio de Trabajo para este puesto. A estos salarios se les incluyen las cargas sociales (16.32\%) correspondientes al seguro social, aguinaldo, vacaciones y cesantía. Otros gastos dentro de la mano de obra incluyen al biólogo asesor de la finca y los eventuales en cosechas y en otras labores (chapeas) de la finca.

En los ciclos cortos se utilizó motor diésel en los bombeos, mientras que en el largo se usó motor eléctrico. El gasto de diésel fue ligeramente variable entre fincas, dependiendo del tamaño del motor, las características de la bomba, la altura de la cabeza de agua que debe levantar y el tipo de descarga (tubo o cajón). Usualmente, se gastó más diésel en las descargas de tubo que en las de cajón. En términos generales, se gastaron 2801 por hectárea de cultivo en una campaña. El precio por litro de diésel en el mes de diciembre del 2013 fue de \$ 1.24 (RECOPE, 2014). El consumo eléctrico fue relativamente constante en la campaña y solo subió cuando hubo que reponer niveles después de las raleas.

El equipo más caro que se debe reponer ocasionalmente es el motor y la bomba en los puestos de bombeo, cuya vida útil es de 5 años. Un bombeo debidamente acondicionado $\mathrm{y}$ en funcionamiento tiene un valor aproximado de \$ 16000 para fincas como las estudiadas de 25 has. Esto significa que se deprecian en un $20 \%$ por año (\$ 3200 ) o un promedio de $\$ 128$ por hectárea al año. Otro equipo auxiliar con un valor aproximado de \$4 200 que también se deprecia rápidamente con una vida útil de 3 años (\$ 1 400/ año o $\$ 56 /$ ha*año) son los medidores de oxígeno disuelto, balanzas, romanas, mallas de cosecha y de filtros, madera, tablones, atarrayas, tanques de siembra y cosecha, herramientas, entre otros.

\section{RESULTADOS}

Las fluctuaciones de temperatura en las mañanas fueron similares entre los ciclos de cultivo, presentándose los valores más bajos $\left(27.5-29.1^{\circ} \mathrm{C}\right) \mathrm{a}$ 
inicios (febrero) y finales (diciembre) del año, los más altos $\left(32.0^{\circ} \mathrm{C}\right)$ en marzo y un promedio cercano a $30.5^{\circ} \mathrm{C}$ en el resto del año. El oxígeno disuelto en las mañanas fue más bajo en el ciclo largo $(2.26 \mathrm{mg} / \mathrm{l})$ que en los ciclos cortos $(3.32 \mathrm{mg} / \mathrm{l})$, y en ambos casos fue disminuyendo con el avance del cultivo, de manera más evidente en el ciclo largo. La turbidez del agua fluctuó de 35 a $50 \mathrm{~cm}$ en el ciclo largo y de 40 a $60 \mathrm{~cm}$ en los ciclos cortos.

Los rendimientos de producción se presentan en el Cuadro 1. Se incluyen los 3 ciclos cortos con sus respectivos totales y promedios $( \pm$ desviación estándar), así como el ciclo largo en el 2013. La variación de los principales parámetros que determinan los rendimientos de producción entre los 3 ciclos cortos consecutivos con respecto al ciclo largo del 2013 fue de un aumento en la supervivencia $(72 \%$ y $63 \%)$ y la tasa de crecimiento $(0.98$ y 0.76 g/semana) y una reducción en la conversión alimenticia $(1.07$ y $1.60: 1.00)$ y la tasa diaria de alimentación (9.55 y $15.86 \mathrm{~kg} / \mathrm{ha} /$ día $)$. La menor supervivencia en el ciclo largo con respecto a los ciclos cortos hizo que la densidad final del cultivo fuera similar a la de siembra en los ciclos cortos. El peso promedio final de las cosechas fue de $13.2 \mathrm{~g}$ en los ciclos cortos y 22.9 g como promedio ponderado, tomando en cuenta las raleas, en el ciclo largo.

En los 3 ciclos cortos, la productividad fue superior $(2605 \mathrm{~kg}$ / ha/año) a la del ciclo largo (2 $135 \mathrm{~kg} /$ ha/año). No obstante, el rendimiento de producción en términos de kilos producidos por cada millar de PL's sembradas fue superior en el ciclo largo (14.4 kg/millar) que en los 3 ciclos cortos consecutivos $(9.2 \mathrm{~kg} /$ millar$)$, indicando un mejor aprovechamiento de las PL's sembradas.

El precio al pie de finca establecido para los diferentes intervalos de tamaños del camarón entero se presenta en el Cuadro 2. Hubo un incremento considerable de los precios en junio del 2013 (>150\% en todas las tallas) en relación con el 2012, debido a la reducción de la oferta internacional del producto en el mercado.

El cálculo de los costos variables de producción más importantes en el ciclo largo y los 3 ciclos cortos del 2013 se presentan en el Cuadro 3 . Estos costos fueron más altos en los ciclos cortos (\$ 8 389) que en el ciclo largo (\$ 6 783). El bombeo con motores diésel aumentó $(12 \%)$ considerablemente el gasto con respecto al eléctrico en el ciclo largo (7\%) no solo por concepto del costo del combustible, sino también por el mantenimiento y lubricación del motor. El rubro que tuvo el mayor incremento del gasto fue el de materiales, equipos e insumos (12\%), sobre todo por concepto del transporte entre las 3 fincas que se encuentran separadas a grandes distancias (Abangaritos de Puntarenas, Colorado de Abangares y Puerto Jesús de Nicoya) entre ellas. La finca en el ciclo largo está localizada en una misma área (Lepanto de Puntarenas), por lo que el gasto por transporte se redujo considerablemente $(6 \%)$. 

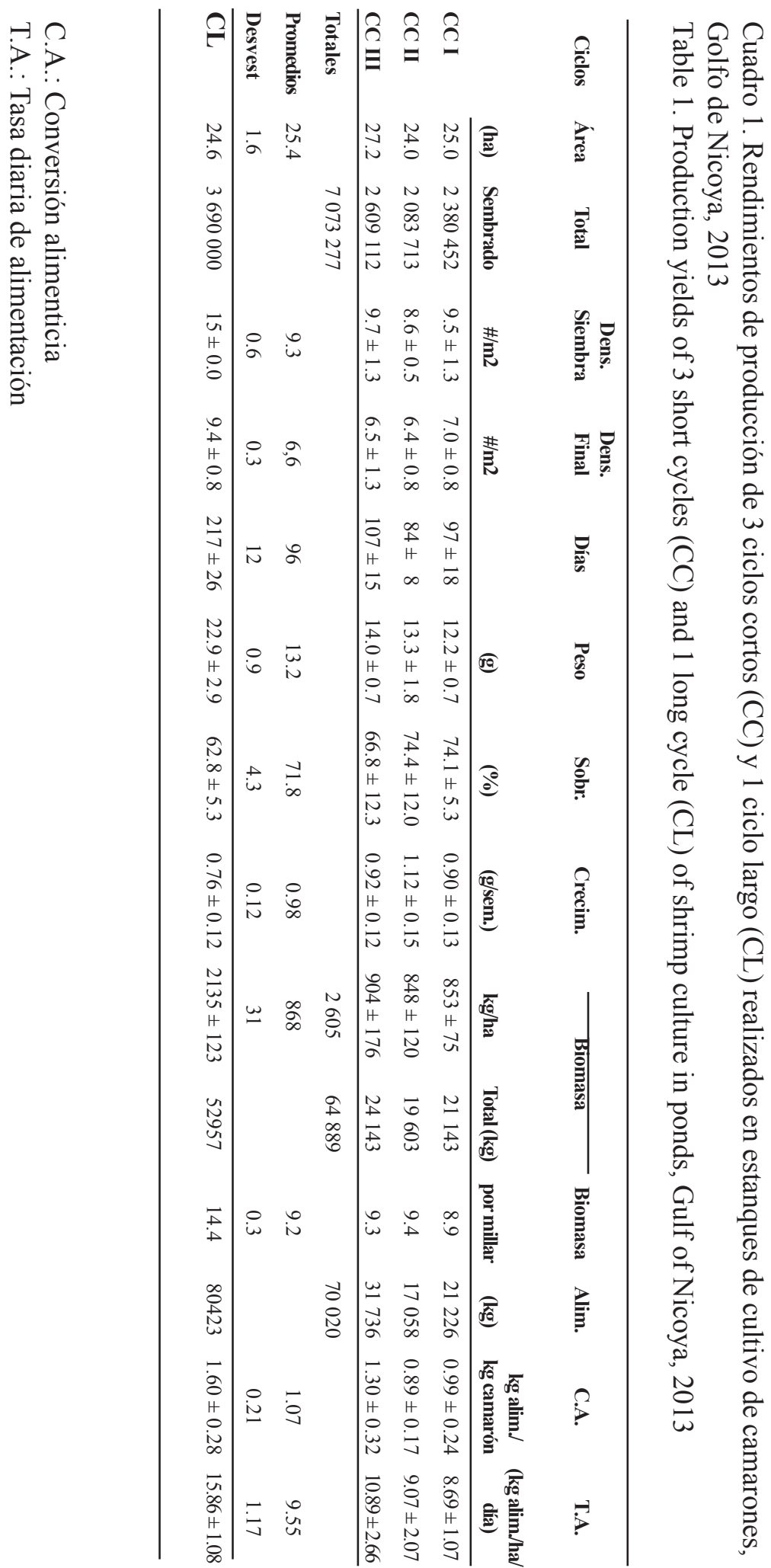
Cuadro 2. Rango de pesos (g), precios por kilo ( $\varnothing$ y US\$) e incremento de precio (\% y precio/g) del camarón a la cosecha en noviembre del 2012 y junio del 2013

Table 2. Weight range (g), prices per $\mathrm{kg}(\phi$ and US\$) and price increment ( $\%$ and price/g) for shrimp harvested in November 2012 and June 2013

\begin{tabular}{cccccccc}
\hline Tamaño & \multicolumn{2}{c}{ Nov. 2012 } & \multicolumn{2}{c}{ Jun. 2013 } & \multicolumn{2}{c}{ Incremento } \\
\hline (g) & $\boldsymbol{c}$ & US\$ & $\boldsymbol{c}$ & US\$ & $\mathbf{( \% )}$ & US\$/g & $\boldsymbol{c} / \mathbf{g}$ \\
$10-11$ & 1100 & 2.20 & 2500 & 5.00 & 227 & 0.27 & 133.3 \\
$11-12$ & 1200 & 2.40 & 2600 & 5.20 & 217 & 0.24 & 121.7 \\
$12-13$ & 1300 & 2.60 & 2700 & 5.40 & 208 & 0.22 & 112.0 \\
$13-14$ & 1400 & 2.80 & 2800 & 5.60 & 200 & 0.21 & 103.7 \\
$14-15$ & 1650 & 3.30 & 2850 & 5.70 & 173 & 0.17 & 82.8 \\
$15-16$ & 1750 & 3.50 & 3000 & 6.00 & 171 & 0.16 & 80.6 \\
$16-17$ & 1850 & 3.70 & 3100 & 6.20 & 168 & 0.15 & 75.8 \\
$17-18$ & 1950 & 3.90 & 3150 & 6.30 & 162 & 0.14 & 68.6 \\
$18-20$ & 2000 & 4.00 & 3200 & 6.40 & 160 & 0.13 & 63.2 \\
$20-21$ & 2100 & 4.20 & 3300 & 6.60 & 157 & 0.12 & 58.5 \\
$21-22$ & 2400 & 4.80 & 3400 & 6.80 & 142 & 0.09 & 46.5 \\
$22-24$ & 2500 & 5.00 & 3900 & 7.80 & 156 & 0.12 & 60.9 \\
$24-25$ & 2600 & 5.20 & 4000 & 8.00 & 154 & 0.11 & 57.1 \\
$25-26$ & 2650 & 5.30 & 4100 & 8.20 & 155 & 0.11 & 56.9 \\
$26-27$ & 2700 & 5.40 & 4300 & 8.60 & 159 & 0.12 & 60.4 \\
$27-28$ & 2750 & 5.50 & 4350 & 8.70 & 158 & 0.12 & 58.2 \\
$28-29$ & 2750 & 5.50 & 4375 & 8.75 & 159 & 0.11 & 57.0 \\
$29-30$ & 2750 & 5.50 & 4500 & 9.00 & 164 & 0.12 & 59.3 \\
$30-32$ & 2800 & 5.60 & 4530 & 9.06 & 162 & 0.11 & 55.8 \\
$32-36$ & 3000 & 6.00 & 5000 & 10.0 & 167 & 0.12 & 58.8 \\
\hline
\end{tabular}

La poslarva también representó un gasto mayor en los ciclos cortos (16\%) por tener que realizarse 3 siembras con el doble de cantidad de poslarva en lugar de una como en el ciclo largo $(12 \%)$. El incremento en el gasto no fue tan considerable, porque se redujo el precio unitario al compartir el flete desde Nicaragua con otras fincas en la importación de mayor cantidad de larvas.

La mano de obra fija fue similar entre las fincas (25\% y $27 \%$ ), utilizando de 4 a
5 hombres para 25 has (5-6 hectáreas por hombre), aunque la vigilancia fue mayor en los ciclos cortos por la necesidad de tener que utilizar un guarda en cada finca. El único rubro más alto en el ciclo largo fue el alimento (46\%) por haberse obtenido una conversión alimenticia mayor (1.6:1.0) que en los ciclos cortos (32\% y 1.1:1.0, respectivamente).

La producción en las cosechas de los ciclos cortos y las raleas o cosechas parciales en el ciclo largo, la 
Cuadro 3. Costos variables de producción (US\$/ha) y respectiva contribución de cada rubro en el cultivo de camarones marinos en un solo ciclo largo y 3 ciclos cortos consecutivos, Golfo de Nicoya, 2013

Table 3. Variable costs of production (US\$/ha) and respective contribution of each category of shrimp culture under 1 long cycle and 3 consecutive short cycles, Gulf of Nicoya, 2013

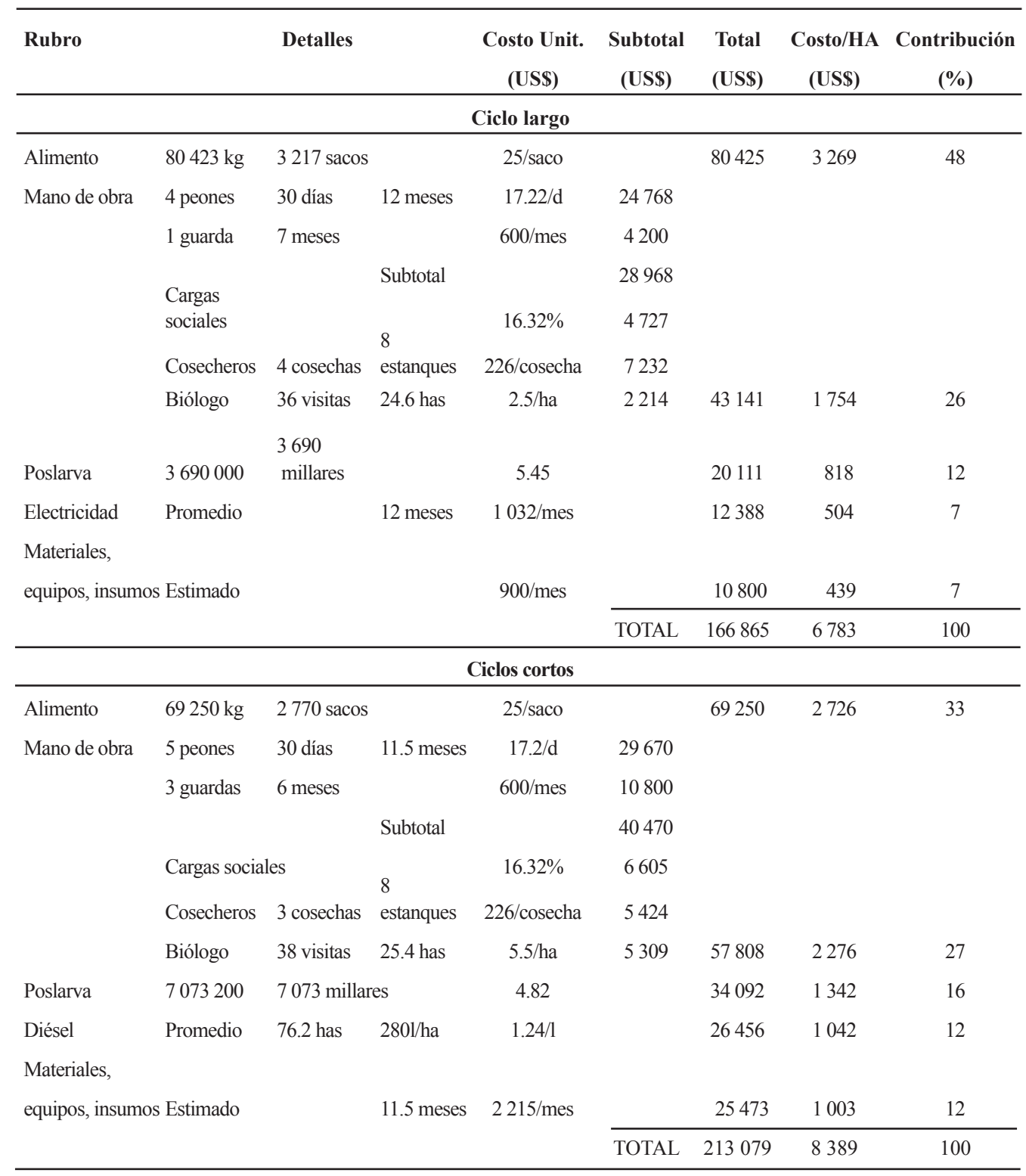

proporción de cada una con respecto al total cosechado, la productividad, el crecimiento del camarón para alcanzar el tamaño de mercado y el precio del producto en el mercado nacional e internacional determinaron los ingresos por concepto de las ventas al pie de finca como se muestra en el Cuadro 4. 
En términos generales, los ciclos cortos generaron más ingresos (\$14 $671 / \mathrm{ha}$ ) por concepto de ventas que el ciclo largo (\$ $12281 / \mathrm{ha}$ ), con una diferencia de US\$ $2390 /$ ha, equivalente en moneda nacional a $\varnothing 1195$ 000/ha en el 2013. Esto significa que en las 25 has estudiadas hubo una superioridad en ventas de \$59 750 o ф 29875000 en los ciclos cortos sobre el ciclo largo. La superioridad fue debida sobre todo a una mayor producción por unidad de área, un crecimiento más alto y un mejor precio del producto por unidad de peso (g) en el mercado nacional. En los ciclos cortos, la producción estuvo entre $850 \mathrm{y}$ $900 \mathrm{~kg} / \mathrm{ha}$, mientras que en el ciclo largo fue muy baja (380-512 kg/ha) en las primeras 3 raleas y solo se emparejó en la cosecha final $(814 \mathrm{~kg} / \mathrm{ha})$.

El crecimiento promedio en los ciclos cortos fue de $0.98 \mathrm{~g} / \mathrm{semana}$ en 96 días, mientras que en el ciclo largo fue más bajo ( $0.85 \mathrm{~g} /$ semana) en un periodo similar de cultivo (99 días). Después mejoró levemente por la influencia de la reducción de la biomasa en las raleas, pero solo llegó a superar el de los ciclos cortos en las últimas semanas de cultivo.

Las ganancias por $\mathrm{kg}$ de producto se establecieron según el precio de venta y los costos de producción. El precio en los ciclos cortos fue de $\$ 5.63 / \mathrm{kg}$ (\$14 671/2 $605 \mathrm{~kg}$ ) y el costo fue de $\$ 3.22 / \mathrm{kg}(\$ 8$ $389 / 2605 \mathrm{~kg}$ ), por lo que la ganancia fue de $\$ 2.41 / \mathrm{kg}$. En el ciclo largo, el precio ponderado fue de $\$ 5.74 / \mathrm{kg}$ ( $\$ 12281 / 2$ $138 \mathrm{~kg}$ ) y el costo fue de $\$ 3.17 / \mathrm{kg}$ (\$6 $783 / 2138 \mathrm{~kg}$ ), siendo la ganancia de $\$$ $2.57 / \mathrm{kg}$. Al ser más alta la productividad en los ciclos cortos, las ganancias (\$6 $278 /$ ha) fueron mayores que las del ciclo largo (\$ 5 495/ha), considerando solo los costos variables de producción.

En el Cuadro 5 se presentan la productividad ( $\mathrm{kg} / \mathrm{ha} / \mathrm{año}), \quad$ los ingresos por concepto de ventas al pie de finca, la depreciación, el costo fijo y las utilidades brutas, sin considerar el pago del impuesto de ventas, para los

Cuadro 4. Variables que determinaron los ingresos por concepto de las ventas de camarón en los 3 ciclos cortos consecutivos y el ciclo largo en el 2013

Table 4. Variables determining income from shrimp sales, using 3 consecutive short cycles and 1 long cycle during 2013

\begin{tabular}{|c|c|c|c|c|c|c|c|c|c|c|}
\hline \multirow[b]{2}{*}{ Cosechas } & \multicolumn{3}{|c|}{ Ciclos cortos } & \multicolumn{7}{|c|}{ Ciclo largo } \\
\hline & $\mathbf{I}$ & II & III & Totales & raleas & $\mathbf{I}$ & II & III & IV & Totales \\
\hline Producción (kg) & 21143 & 19603 & 24143 & 64889 & & 12510 & 10915 & 6649 & 22464 & 52538 \\
\hline Proporción del total (\%) & 33 & 30 & 37 & 100 & & 24 & 21 & 13 & 43 & 100 \\
\hline Productividad (kg/ha) & 853 & 848 & 904 & 2605 & & 512 & 431 & 381 & 814 & 2138 \\
\hline Tamaño promedio (g) & 12.2 & 13.3 & 14.0 & & & 11.8 & 18.4 & 25.1 & 29.5 & \\
\hline Duración (días) & 97 & 84 & 107 & & & 99 & 141 & 180 & 210 & \\
\hline Crecimiento (g/semana) & 0.90 & 1.12 & 0.92 & & & 0.85 & 0.92 & 0.98 & 1.00 & \\
\hline Precio promedio (US\$) & 5.49 & 5.60 & 5.80 & & & 4.39 & 5.65 & 7.03 & 8.70 & \\
\hline Precio unidad (US\$/g) & 0.45 & 0.42 & 0.41 & & & 0.37 & 0.31 & 0.28 & 0.29 & \\
\hline Ventas (US\$/ha) & 4679 & 4749 & 5243 & 14671 & & 2252 & 2421 & 2685 & 4923 & 12281 \\
\hline
\end{tabular}


3 ciclos cortos consecutivos y el ciclo largo del 2013.

Las utilidades brutas fueron mayores en los 3 ciclos cortos consecutivos que en un solo ciclo largo en el 2013 con una superioridad de \$ 784/ ha. Esto significa que en fincas con un promedio de 25 has como las estudiadas, los ciclos cortos generaron \$ 19 600 más que un ciclo largo, lo que en moneda nacional significa $\varnothing 9800000$ adicionales al año.

El análisis de sensibilidad económica también se puede establecer en términos del punto de equilibrio, el cual indica el valor del producto (costo total de producción/producción) y el nivel de producción (costo total de producción/ precio por kilo) en el que los ingresos apenas alcanzan para cubrir los costos. En el primer caso, el valor por kg de producto en equilibrio en el ciclo corto es en promedio de $\$ 3.22(\$ 8389 / 2605 \mathrm{~kg})$ y en el ciclo largo es de $\$ 3.17$ (\$ $6783 / 2$ $138 \mathrm{~kg}$ ) como promedio ponderado. En el segundo caso, el nivel de producción en equilibrio es de $1490 \mathrm{~kg} / \mathrm{ha} /$ año $(\$ 8$ $389 / \$ 5.63)$ en los ciclos cortos y 1182 $\mathrm{kg} / \mathrm{ha} / \mathrm{año}(\$ 6783 / \$ 5.74)$ en el ciclo largo. El análisis indica que con el 55-57\% de lo cosechado, que corresponde a 1.75 cosechas en los ciclos cortos y 3 raleas en el ciclo largo, se pagan los costos de operación y quedan las ganancias antes del pago de impuestos, la depreciación y los costos fijos.

\section{DISCUSIÓN}

La temperatura del agua en las mañanas del 2013 presentó valores similares a los del 2012; sin embargo, no bajó de $29^{\circ} \mathrm{C}$, mientras que en el 2012 bajó a $27^{\circ} \mathrm{C}$ (Valverde-Moya \& Alfaro-Montoya, 2013). Este fenómeno se explica porque en octubre del 2013 aún dominaba una condición neutral con tendencia leve de calentamiento en todo el Pacífico ecuatorial (Alvarado, 2013). La supervivencia, el crecimiento y los rendimientos de producción se han reducido en otros países latinoamericanos, debido a las bajas temperaturas ambientales y del agua por efecto de los frentes fríos en esta época (Scura, 1995; Ponce-Palafox et al. 1997; Krummenauer et al. 2010).

El oxígeno disuelto, en el ciclo largo, registró niveles inferiores de 3.0 $\mathrm{mg} / \mathrm{l}$ durante 27 semanas, sin afectar los rendimientos de producción. Sin

Cuadro 5. Productividad, ingresos totales, costos variables, depreciación y costos fijos que determinaron la rentabilidad del cultivo de camarones marinos en 3 ciclos cortos y 1 solo ciclo largo, Golfo de Nicoya, 2013

Table 5. Productivity, total income, variable costs, depreciation and fixed costs that determined shrimp culture profitability under 3 short cycles and 1 long cycle, Gulf of Nicoya, 2013

\begin{tabular}{lcccccc}
\hline Ciclos & $\begin{array}{c}\text { Productividad } \\
\text { (kg/ha/año) }\end{array}$ & $\begin{array}{c}\text { Ingresos } \\
\text { (US\$/ha) }\end{array}$ & $\begin{array}{c}\text { Costos } \\
\text { variables } \\
\text { (US\$/ha) }\end{array}$ & $\begin{array}{c}\text { Depreciación } \\
\text { (US\$/ha) }\end{array}$ & $\begin{array}{c}\text { Costos fijos } \\
\text { (US\$/ha) }\end{array}$ & $\begin{array}{c}\text { Rentabilidad } \\
\text { (US\$/ha) }\end{array}$ \\
\hline Cortos & 2605 & 14671 & 8389 & 184 & 562 & 5536 \\
Largo & 2138 & 12281 & 6783 & 184 & 562 & 4752 \\
\hline
\end{tabular}


embargo, los ciclos cortos con niveles de oxígeno sobre $3.0 \mathrm{mg} / 1$ mostraron mejor crecimiento, consumo de alimento y conversión alimenticia. Con las condiciones propias de cultivo en el Golfo de Nicoya, se puede establecer un nivel óptimo o aceptable de $3.0 \mathrm{mg} / \mathrm{l}$ para no afectar el crecimiento ni la conversión alimenticia, un nivel crítico de 2.0 $\mathrm{mg} / \mathrm{l}$ que no afecta la sobrevivencia, pero resulta estresante para el camarón pudiendo influir negativamente en el crecimiento (Kramer, 1975) y un nivel letal cercano a $1.0 \mathrm{mg} / \mathrm{l}$ (Martínez et al. 1998) que causa flotamiento $\mathrm{y}$ mortalidad del camarón.

La mayor supervivencia (74\%) en los 2 primeros ciclos cortos con respecto al ciclo largo (63\%) revela la relación inversa que se ha encontrado en otros estudios entre sobrevivencia y densidad de siembra. El tercer ciclo corto del año presentó una sobrevivencia inferior (67\%), que se explica por la transición entre la época lluviosa y la seca (Valverde-Moya \& Alfaro-Montoya, 2013). En términos generales, el rango de supervivencia (63-75\%) en todos los ciclos fue similar al obtenido en Venezuela luego de 11 generaciones de 1990 al 2001 (59 a 76\%) y ligeramente superior a los valores reportados (58 a $65 \%$ ) en otros estudios, pero con densidades superiores de siembra (17 a $45 / \mathrm{m}^{2}$; Sookying et al. 2011).

El mayor crecimiento (0.98 g/ semana) en los ciclos cortos en relación con el ciclo largo (0.76 g/ semana) también pudo ser debido a una influencia positiva de la menor densidad de siembra, como se ha demostrado en otros estudios (Sookying et al. 2011). Sin embargo, la tasa de crecimiento en el ciclo largo fue similar a las obtenidas $(0.76$ a 0.87 $\mathrm{g} /$ semana) en estanques grandes en Venezuela desde 1990 hasta el 2002 con densidades de siembra parecidas (De Donato et al. 2005).

Las conversiones alimenticias obtenidas en los 2 primeros ciclos cortos con valores inferiores a 1.0 demostraron un estricto manejo de la alimentación. Una conversión alimenticia de 1.6:1.0 como la obtenida en el ciclo largo puede considerarse excesiva para nuestras condiciones propias de manejo, aunque está dentro del rango aceptable (1.5-2.4:1.0) en otros países como Venezuela y Brasil con densidades similares de cultivo (De Donato et al. 2005; Krummenauer et al. 2010).

La productividad definida en términos de $\mathrm{kg} / \mathrm{ha} / \mathrm{año}$ fue superior en los 3 ciclos cortos $(2605 \mathrm{~kg} / \mathrm{ha} /$ año) con respecto al ciclo largo (2 $138 \mathrm{~kg} / \mathrm{ha} / \mathrm{año})$. Estas productividades son superiores a las obtenidas en Brasil (1 $962 \mathrm{~kg} / \mathrm{ha}$ en 2 ciclos cortos consecutivos de 75 días cada uno y $1322 \mathrm{~kg} / \mathrm{ha}$ en un ciclo largo de 150 días) con una densidad de siembra de $10 / \mathrm{m}^{2}$ (Krummenauer et al. 2010) y en Texas (1 $777 \mathrm{~kg} / \mathrm{ha} / \mathrm{año})$ en condiciones semiintensivas de cultivo (Samocha et al. 2004). Son similares a las obtenidas en Alabama con una densidad de siembra de $17 / \mathrm{m}^{2}$ (2 660 $\mathrm{kg} / \mathrm{ha}$; Sookying et al. 2011). 
Los precios del camarón pequeño (10-14 g) para el mercado nacional tuvieron enormes incrementos durante el 2012-2013 (200\%). Esto fue debido a que países vecinos como Nicaragua, Honduras y Panamá, de donde se importaba anteriormente, dedicaron la mayor parte de la producción a la exportación hacia México, Estados Unidos y Europa, debido a la reducción en la oferta y al incremento considerable en el precio. Datos de Nicaragua reflejan que hasta octubre del 2013, las exportaciones de camarón habían ascendido a $\$ 75.6$ millones con un aumento del $12 \%$, con respecto al valor total del 2012 que fue de \$ 67.5 millones (El nuevo diario.com.ni, 2013). La industria camaronera en Honduras cerró el 2013 con ingresos de $\$ 190$ millones por exportaciones, \$22.8 millones más que al cierre del año anterior (Centra1AmericaData.COM, 2013c). Durante los primeros tres meses del 2013, la actividad pesquera fue la que reportó mayor crecimiento económico en $\mathrm{Pa}$ namá, sumando $\$ 38.7$ millones frente a los $\$ 28.2$ millones reportados en el 2012 , y de ella, el $88 \%$ de aumento en las ventas lo tuvieron los camarones (CentralAmericaData.COM, 2013d).

En el cálculo de la depreciación se tomó en cuenta que las fincas analizadas en este estudio fueron construidas a inicios de los noventa, al igual que las demás en la zona norte del país, por lo que tienen más de 20 años de uso, habiendo cumplido ya su vida útil en cuanto a estanques, canales, compuertas, tuberías, bodegas, entre otros aspectos. Hasta el momento no se prevé que se vayan a hacer mejoras significativas en estas infraestructuras, debido a que aún se conservan en buen estado de funcionamiento.

Los ingresos por concepto de ventas al pie de finca fueron más altos en los ciclos cortos (\$14 671/ha) que en el ciclo largo (\$ 12 281/ha). Estos dependieron exclusivamente del precio del producto en el mercado. Probablemente, el productor en el ciclo largo asumió que su principal mercado y el que le iba a dar las mayores ganancias económicas era el de exportación, por lo que prefirió vender más barato al mercado nacional con tal de alcanzar las tallas grandes que necesitaba para exportar. El análisis fue hecho en el 2013 cuando los precios, sobre todo del camarón pequeño para el mercado nacional, subieron hasta en un $200 \%$. Si el mismo análisis se hubiera hecho en el 2012 cuando los precios fueron bajos, los ingresos por ventas habrían sido de $\$ 7$ 123/ha en los ciclos cortos y de $\$ 9683 /$ ha en el ciclo largo.

El número de ciclos cortos se puede incrementar hasta en 4 o más por año con el uso de precrías, una práctica sugerida en este estudio y que se está haciendo común en las fincas camaroneras del Golfo de Nicoya. En algunas fincas del Golfo de Nicoya se utiliza una etapa intermedia entre la precría y el estanque de engorde estableciendo un sistema de maternidad $\left(75 / \mathrm{m}^{2}\right)$, desarrollo o preengorde $(10 /$ $\mathrm{m}^{2}$ ) y engorde $\left(6 / \mathrm{m}^{2}\right)$ (Biol. Fernando Vives, comunicación personal).

Otra posibilidad de incrementar la producción de camarón pequeño es intensificando los cultivos por medio del 
aumento en las densidades de siembra a 25 camarones $/ \mathrm{m}^{2}$ y el uso de aireadores. Sin embargo, cultivos intensivos no han sido prácticos en nuestro país después de varios intentos hasta la fecha por la elevada inversión, los altos costos de producción y el eminente riesgo de pérdidas económicas (Krummenauer et al. 2010). Modelos de simulación han detectado que $L$. vannamei en una densidad de $13.5 / \mathrm{m}^{2}$ ha dado los mayores rendimientos de producción en términos de peso final, productividad, consumo de alimento, principales costos de operación (alimento y poslarva) y utilidad económica en una sola cosecha por año (Pardy et al. 1983). Por eso, la mayoría de productores prefieren los sistemas semiintensivos de cultivo en América Latina. Aunque un estudio en Honduras indicó que a mayor densidad se aumenta la productividad y rentabilidad del cultivo en la estación lluviosa, en el largo plazo se ha preferido una producción más sostenible sembrando a bajas densidades sobre todo por la reducción de los desechos metabólicos que deterioran los fondos (TeichertCoddington \& Rodríguez, 1995).

Otra alternativa futura para mejorar la producción es el cultivo monosexual de poslarvas $100 \%$ hembras, que presentan una mayor tasa de crecimiento que los machos, en subadultos y adultos. En L. vannamei, el dimorfismo sexual de tamaño comienza a los $10 \mathrm{~g}$ (Chow \& Sandifer, 1991) y se hace significativo alrededor de los $17 \mathrm{~g}$ (Pérez-Rostro \& Ibarra, 2003), que es la talla convencional de cosecha (Campos-Ramos et al. 2006).
Esta posibilidad tecnológica ha sido desarrollada para langostinos de agua dulce, Macrobrachium rosenbergii (Ventura \& Sagi, 2012), con ventajas comerciales demostradas; sin embargo, en camarones marinos se requiere de investigación científica y evaluación económica.

El estudio de sensibilidad económica sugiere la realización de 3 ciclos cortos en lugar de un solo ciclo largo, debido a la mayor productividad (2 $605 \mathrm{~kg} / \mathrm{ha} / \mathrm{año}$ contra $2138 \mathrm{~kg} / \mathrm{ha} / \mathrm{año}$ ) y rentabilidad (\$ 5 536/ha contra $\$ 4752 / \mathrm{ha}$ ). Un estudio en Brasil con una densidad de siembra de 10 camarones $/ \mathrm{m}^{2}$ dio una rentabilidad inferior, la cual fue de $\$ 3807 /$ ha en 2 ciclos cortos y \$ 3902 en un solo ciclo largo en el 2010. Análisis de sensibilidad económica (Hatch et al. 1987) han demostrado que las estrategias de manejo han sido más sensibles al costo del alimento y los cambios en el precio del camarón que al costo de la poslarva y de la energía (eléctrica o diésel). Con respecto a la poslarva, ha sido más importante su disponibilidad y calidad que el precio por la relación costo: beneficio.

\section{CONCLUSIONES}

Con el análisis de estos costos, el productor debe ser capaz de tomar decisiones y hacer ajustes en el sistema de cultivo para adaptarlo a sus posibilidades económicas haciéndolo más eficiente. En los ciclos cortos, por ejemplo, puede reducir los costos por combustible mejorando el sistema de bombeo, el de transporte utilizando un camión más grande para hacer menos viajes y el de poslarva haciendo pedidos más grandes y compartidos para reducir 
el flete. En el ciclo largo hay que reducir el gasto excesivo por concepto del alimento haciendo ajustes en la tabla de alimentación, las frecuencias alimenticias, la estimación de la biomasa, el uso de comederos o indicadores y la pericia de los alimentadores en la aplicación del alimento diario.

La estrategia de producción no debe ser fija, sino flexible para ajustarse al cambio, sobre todo del precio del producto en el mercado en esos momentos. Cuando los precios nacionales para el camarón pequeño son bajos por la importación de camarón u otra variable, conviene más llevarlo a tallas grandes para la exportación haciendo un solo ciclo largo o alternando un ciclo corto y uno largo como lo recomendaron ValverdeMoya \& Alfaro-Montoya (2013). Por el contrario, cuando hay poco camarón y los precios son altos en el mercado nacional, lo más conveniente es hacer tantos ciclos cortos como sean posibles.

Las producciones en el punto de equilibrio fueron inferiores a las obtenidas al final del cultivo en cada caso, indicando que con un $56 \%$ como promedio de cosechado se pagan los costos de operación. A pesar de que estos resultados son positivos y optimistas, se sugiere mucha precaución para el productor a la hora de decidirse por uno u otro tipo de cultivo, porque cada año hay que tomar en cuenta las condiciones climáticas, los costos de producción, la oferta, la demanda y, sobre todo, el precio del producto en el mercado tanto nacional como internacional.
Independientemente del ciclo que se escoja, hay que tratar de ser lo más eficientes posibles buscando y adaptando estrategias que aumenten la productividad, reduzcan los costos de producción, le den valor agregado al producto, suban el precio y generen las mayores ganancias económicas.

\section{AGRADECIMIENTOS}

Los autores agradecen la cooperación de los productores en las fincas camaroneras ubicadas en las comunidades de Colorado y Jicaral, a ambas márgenes del Golfo de Nicoya. Un particular agradecimiento a los evaluadores por sus valiosas observaciones.

\section{BIBLIOGRAFÍA}

Alvarado, L. (2013). Fase del fenómeno... neutral...condición actual del fenómeno ENOS. B. ENOS, 57, 1-7.

Álvarez, J. \& Salazar, E. (2010). La pesca de arrastre en Costa Rica. Recuperado en diciembre 13, 2013, disponible en http://www.marviva.net/Publicaciones/ PESCA ARRASTRE CR.pdf

Campos-Ramos, R., Garza-Torres, R., Guerrero-Tortolero, D. A., MaedaMartínez, A. M. \& Obregón-Barboza, H. (2006). Environmental sex determination, external sex differentiation and structure of the androgenic gland in the Pacific white shrimp Litopenaeus vannamei (Boone). Aquac. Res., 37(15), 1583-1593. DOI: http://dx.doi. org/10.1111/j.1365-2109.2006.01604.x

Castillo, D. (2012). Sector camaronero busca dinamizar exportaciones. Recuperado en diciembre 13, 2013, disponible en http://www.crhoy.com/sector-camaronero-busca-dinamizar-exportaciones/ 
CentralAmericaData.COM. (2012). Tendencias del consumidor de camarón. Recuperado en diciembre 14, 2014, disponible en http://m.centralamericadata.com/es/article/home/Tendencias del consumidor de camaron

CentralAmericaData.COM. (2013a). Oportunidades en el mercado del camarón. Recuperado en diciembre 15, 2013, disponible en http://www. centralamericadata.com/es/article/home/ Oportunidades en el mercado del camaron CentralAmericaData.COM. (2013b). Costa Rica prohíbe redes de arrastre en pesca de camarón. Recuperado en diciembre 15, 2013, disponible en http:// www.centralamericadata.com/es/article/home/Costa Rica prohíbe redes de arrastre en pesca de camaron

CentralAmericaData.COM. (2013c). Balance 2013 de la industria camaronera de Honduras. Recuperado en diciembre 15, 2013, disponible en http://www. centralamericadata.com/es/article/home/ Balance 2013 de la industria camaronera de Honduras 2013

CentralAmericaData.COM.

(2013d).

Se reactiva el sector pesquero de Panamá. Recuperado en diciembre 16, 2013, disponible en http://www. centralamericadata.com/es/article/home/ Se reactiva el sector pesquero de Panama Chow, S. \& Sandifer, P. A. (1991). Differences in growth, morphometric traits and male sexual maturity among Pacific white shrimp, Penaeus vannamei, from different commercial hatcheries. Aquaculture, 92, 165-178. DOI: http://dx.doi. org/10.1016/0044-8486(91)90018-3

De Donato, M., Manrique, R., Ramírez, R., Mayer, L. \& Howell, C. (2005). Mass selection and inbreeding effects on a cultivated strain of
Penaeus (Litopenaeus) vannamei in Venezuela. Aquaculture, 247(2), 159167. DOI: http://dx.doi.org/10.1016/j. aquaculture.2005.02.005

El nuevo diario.com.ni. (2013). Exportación ha crecido $12 \%$ este año. Recuperado en diciembre17,2013, disponibleenhttp://www. elnuevodiario.com.ni/economia/304058mas-camaron-nica-al-exterior

Hatch, U., Sindelar, S., Rouse, D. \& Pérez, H. (1987). Demonstrating the use of risk programming for aquacultural farm management: The case of penaeid shrimp in Panama. J. World Aquac. Soc., 18(4), 260-269. DOI: http://dx.doi. org/10.1111/j.1749-7345.1987.tb01037.x

Kramer, G. L. (1975). Studies on the lethal dissolved oxygen levels for young brown shrimp, Penaeus aztecus Ives. Proc. World Maric. Soc., 6(4), 157-167.

Krummenauer, D., Cavalli, R. O., Ballester, E. L. C. \& Wasielesky, W. Jr. (2010). Feasibility of Pacific white shrimp Litopenaeus vannamei culture in southern Brazil: effects of stocking density and a single or a double CROP management strategy in earthen ponds. Aquac. Res., 41(2), 240-248. DOI: http://dx.doi. org/10.1111/j.1365-2109.2009.02326.x Martínez, E., Aguilar, M., Trejo, L., Hernández, I., Díaz-Iglesia, E., Soto, L. A., Sánchez, A. \& Rosas, C. (1998). Lethal low dissolved oxygen concentrations for postlarvae and early juvenile Penaeus setiferus at different salinities and pH. J. World Aquac. Soc., 29(2), 221-229. DOI: http:// dx.doi.org/10.1111/j.1749-7345.1998. tb00980.x

Ministerio de Trabajo y Seguridad Social. (2013). Lista de salarios mínimos: segundo semestre, 2013. Recuperado en diciembre 21, 2013, disponible en http:// 
www.tusalario.org/costarica/documents/ salarios-minimos-ii-semestre

Pardy, C. R., Griffin, W. L., Johns, M. A. \& Lawrence, A. L. (1983). A preliminary economic analysis of stocking strategies for penaeid shrimp culture. J. World Maric. Soc., 14(1-4), 49-63. DOI: http://dx.doi. org/10.1111/j.1749-7345.1983.tb00059.x

Pérez-Rostro, C. I. \& Ibarra, A. M. (2003). Heritabilities and genetic correlations of size traits at harvest size in sexually dimorphic Pacific white shrimp (Litopenaeus vannamei) grown in two environments. Aquac. Res., 34, 1079-1085. DOI: http://dx.doi.org/10.1046/j.13652109.2003.00913.x

Ponce-Palafox, J., Martínez-Palacios, C. \& Ross, L. (1997). The effect of salinity and temperature on the growth and survival rates of juvenile white shrimp, Penaeus vannamei, Boone, 1931. Aquaculture, 157(2), 107115. DOI: http://dx.doi.org/10.1016/ S0044-8486(97)00148-8

PROCOMER, Promotora del Comercio Exterior de Costa Rica. (2013). Crece la competencia en el mercado de camarón. Recuperado en diciembre 21, 2013, disponible en http://www.procomer.com/ contenido/articles/crece-la-competenciaen-el-mercado-de-camar $\% \mathrm{C} 3 \% \mathrm{~B} 3 \mathrm{n} . \mathrm{html}$

RECOPE, Refinería Costarricense de Petróleo S.A. (2014). Precios históricos, enero 1994 a la fecha. Recuperado en mayo 22, 2014, disponible en http://www.recope.go.cr/wp-content/ uploads/2014/05/PRECIOS-HISTORICOS-de-regularsuper-y-diesel-conpescadores-22-05-2014.pdf

Samocha, T. M., López, I. M., Jones, E. R., Jackson, S. \& Lawrence, A. L. (2004).
Characterization of intake and effluent waters from intensive and semi-intensive shrimp farms in Texas. Aquac. Res., 35(4), 321-339. DOI: http://dx.doi. org/10.1111/j.1365-2109.2004.01002.x Scura, E. (1995). Dry season production problems on shrimp farms in Central America and the Caribean basin. En C. Browdy \& J. Hopkins (Eds.), Swimming through troubled waters (pp. 200-213). Luisiana, EE.UU.: World Aquaculture Society.

Sookying, D., Soller, F., Davis, D. A. \& Hanson, T. (2011). Effects of stocking density on the performance of Pacific white shrimp Litopenaeus vannamei cultured under pond and outdoor tank conditions using a high soybean meal diet. Aquaculture, 319(2), 232239. DOI: http://dx.doi.org/10.1016/j. aquaculture.2011.06.014

Teichert-Coddington, D. R. \& Rodríguez, R. (1995). Semi-Intensive commercial grow-out of Penaeus vannamei fed diets containing differing levels of crude protein during wet and dry seasons in Honduras. J. World Aquac. Soc., 26(1), 72-79. DOI: http://dx.doi. org/10.1111/j.1749-7345.1995.tb00211.x Valverde-Moya, J. \& Alfaro-Montoya, J. (2013). La experiencia del cultivo comercial de camarones marinos en estanques de producción en Costa Rica. Rev. Mar. Cost., 5, 87-105.

Ventura, T. \& Sagi, A. (2012). The insulin-like androgenic gland hormone in crustaceans: From a single gene silencing to a wide array of sexual manipulation-based biotechnologies. Biotechnol. Adv., 30(6), 1543-1550. DOI: http://dx.doi.org/10.1016/j. biotechadv.2012.04.008 